\title{
Autonomous Orbit Determination for Formations of Cubesats Beyond LEO
}

\author{
Massimiliano Vasile ${ }^{1, *}$ \\ 75 Montrose Street, Department of Mechanical and Aerospace Engineering, Glasgow, UK \\ Francesco Torre ${ }^{2, *}$ \\ 75 Montrose Street, Department of Mechanical and Aerospace Engineering, Glasgow, UK \\ Romain Serra ${ }^{3, *}$ \\ 75 Montrose Street, Department of Mechanical and Aerospace Engineering, Glasgow, UK \\ Stuart Grey ${ }^{4, *}$ \\ 75 Montrose Street, Department of Mechanical and Aerospace Engineering, Glasgow, UK
}

\begin{abstract}
This paper investigates the use of the Time Of Arrival (TOA) and Doppler shift to allow a small formation of CubeSats to navigate beyond low Earth orbit (LEO). The idea is to use a one way communication, from one or more ground stations to two or more CubeSats, to reconstruct an estimation of the position and velocity of the formation with respect to Earth. The paper considers the use of the difference in TOA and Doppler measurements to mitigate the error introduced by the onboard clock. These measurements are combined with inter-satellite distance and velocity measurements based on a two-way communication between pairs of spacecraft. The paper will provide
\end{abstract}

\footnotetext{
This template can be used for all publications in Advances in Space Research.

* Corresponding author

Email address: massimiliano.vasile@strath.ac.uk (Massimiliano Vasile)

${ }^{1}$ Professor space systems engineering, Aerospace Centre of Excellence, University of Strathclyde

${ }^{2} \mathrm{PhD}$ candidate, Aerospace Centre of Excellence, University of Strathclyde

${ }^{3}$ Research fellow, Aerospace Centre of Excellence, University of Strathclyde

${ }^{4}$ Lecturer, Aerospace Centre of Excellence, University of Strathclyde
} 
an estimation of the error in position and velocity that can be obtained by a combination of these measurements. The reference case for these analyses will be a transfer trajectory to the Moon.

Keywords: cubesat; orbit determination; deep space; TOA;

\section{Introduction}

There is a growing interest in using nanosatellites (like CubeSats) beyond LEO. From the proposed NASA Mars mission MarCO (Asmar \& Matousek (2008)) to more recent mission studies for CubeSats to the Moon and asteroids, the goal is to achieve significant scientific results with small, low-cost and compact spacecraft. The challenge in all these cases is to achieve mission objectives with limited resources.

Given the low cost of the platform some limitations can be mitigated by using more than one nanosatellite working in team and distributing tasks and resources. The paper is proposing a combination of measurements that can be used to autonomously determine position and velocity of CubeSats flying in formation in deep space or in the vicinity of a minor body. The limited power on-board a CubeSat might not be sufficient to implement traditional tracking techniques for orbit determination based on ground support (Differential One-way Range (DOR) or delta-DOR for example). Furthermore, the precision of the clocks on-board a CubeSat might not allow for one way ranging and range rate measurements with sufficient accuracy if no synchronisation strategy is applied.

The paper is investigating the possibility to exploit the inter-satellite link between two, or more CubeSats, together with different combinations of Time Of Arrival (TOA), Time Difference Of Arrival (TDOA) and Frequency Of Arrival (FOA) to estimate position and velocity with respect to a known beacon. The approach is similar to known techniques for the localisation of a source given the known position of different receivers (see Torrieri (1984); Ulman (2001); Ho \& Chan (1997); Mason (2004)) albeit it assumes one or more known ground emitters and unknown space receivers. The signal emitted from one or more ground stations (the known beacons), and received by two or more spacecraft, provides information on time, frequency and location of emission. The time and frequency at which the signal is received depend on the position and velocity of the spacecraft. In general two spacecraft will receive the same signal at different times and with different frequency 
shifts. If the CubeSats can measure, with reasonable accuracy, their relative position and velocity, the knowledge of the relative position between the two spacecraft in combination with the time and frequency difference of the received signal are sufficient to estimate the position and velocity with respect to the known beacon. The paper explores two scenarios: one with two beacons and two receivers and one with a single beacon and three receivers. The analysis is theoretical in that it does not consider real stations or a real mission, though ranges of errors in TOA, FOA and intersatellite links are compatible with current technology.

The paper is organised as follows. First the dynamics and measurement models are introduced, followed by an analytical derivation of the state estimation solution for both the three-spacecraft one station case and the twospacecraft two-station cases. This analysis provides insight into the number of possible solutions as a function of the geometric configuration of the spacecraft and ground stations. Then, some numerical experiments are used to quantify the estimation error. The reference case is a trajectory to the Moon.

\section{Dynamic Model and Formation Design}

The dynamics of the spacecraft is defined in the Earth Centred Inertial reference frame et epoch and subject only to the spherical homogeneous gravity filed of the Earth. In this framework one can approximate the position of the spacecraft in the formation with respect to a given reference orbit as:

$$
\begin{aligned}
\delta x_{r}= & \frac{r}{a} \delta a+\frac{a e \sin \theta}{\sqrt{1-e^{2}}} \delta M-a \cos \theta \delta e \\
\delta y_{\theta}= & \frac{r}{\left(1-e^{2}\right)^{3 / 2}}(1+e \cos \theta)^{2} \delta M+r \delta \omega \\
& +\frac{r \sin \theta}{\left(1-e^{2}\right)}(2+e \cos \theta) \delta e+r \cos i \delta \Omega \\
\delta z_{h}= & r\left(\sin \theta^{*} \delta i-\cos \theta^{*} \sin i \delta \Omega\right)
\end{aligned}
$$

where $\delta \mathbf{r}=\left[\delta x_{r}, \delta y_{\theta}, \delta z_{h}\right]^{T}$ is the relative position vector of a spacecraft in a radial, transversal, out-of-plane reference frame attached to the reference orbit, $\theta$ is the true anomaly along the reference orbit, $\theta^{*}=\theta+\omega, r, a, e, i$ and $\omega$ are respectively the radius, semi-major axis, eccentricity, inclination, argument of the pricentre of the reference orbit, and $\delta a, \delta e, \delta i, \delta \Omega, \delta \omega, \delta M$ are the variations of the orbital parameters. 
By properly setting the values of the variation of the orbital parameters, one can design the desired formation with the required separation among spacecraft. In order to test the orbit determination approach, we propose a formation that rests in the $\theta-h$ plane and that brings the spacecraft periodically at close distance. As it will be demonstrated in the following, this geometry presents no singularities for most of the orbit and allows one to study the sensitivity of the determination error to the inter-satellite distance.

The orbital parameters of the reference orbit are reported in Table 1. In the following we will study two cases: a formation of three spacecraft receiving the signal of a single ground station and a formation of two spacecraft receiving the signal of two ground stations. The $\delta$ elements for the two formations are in Table 2 and the nominal trajectories in the $\theta-h$ plane can be found in Figs. 1 and 2.

Table 1: Orbital elements of the reference trajectory

\begin{tabular}{cccccc}
\hline $\mathrm{a}[\mathrm{km}]$ & $\mathrm{e}$ & $\mathrm{i}[\mathrm{deg}]$ & $\Omega[\mathrm{deg}]$ & $\omega[\mathrm{deg}]$ & $\nu_{0}[\mathrm{deg}]$ \\
\hline 195539 & 0.966 & 7 & 0 & 0 & 0 \\
\hline
\end{tabular}

Table 2: Delta Orbital elements of the two formations

\begin{tabular}{crrrrrr}
\hline \hline & $\delta a[\mathrm{~km}]$ & $\delta e$ & $\delta i[\mathrm{rad}]$ & $\delta \Omega[\mathrm{rad}]$ & $\delta \omega[\mathrm{rad}]$ & $\delta M[\mathrm{rad}]$ \\
\hline 3-s/c formation & & & & & & \\
\hline s/c 1 & 0 & 0 & $4 e-4$ & $-14 e-4$ & $-1 e-4$ & 0 \\
s/c 2 & 0 & 0 & $4 e-4$ & $14 e-4$ & $-2.8 e-3$ & 0 \\
s/c 3 & 0 & 0 & $4 e-4$ & $14 e-4$ & $1 e-4$ & 0 \\
\hline 2-s/c formation & & & & & & \\
\hline s/c 1 & 0 & 0 & $-2 e-3$ & $-4 e-4$ & 0 & 0 \\
s/c 2 & 0 & 0 & $2 e-3$ & $4 e-4$ & 0 & 0 \\
\hline
\end{tabular}

\section{Measurement Model}

In this section we describe the type of measurements considered for the determination of position and velocity of the CubeSats. Four types of measurements are considered: the relative position and velocity via inter-satellite link, the Time Difference of Arrival and the Time Of Arrival of the signal received from one or more beacons and the Doppler shift. 


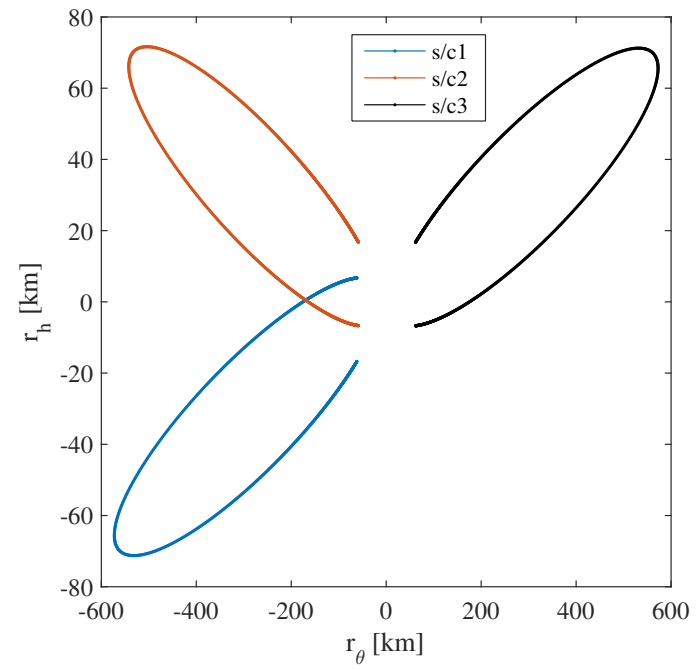

Figure 1: Three spacecraft formation in the $\theta-h$ plane.

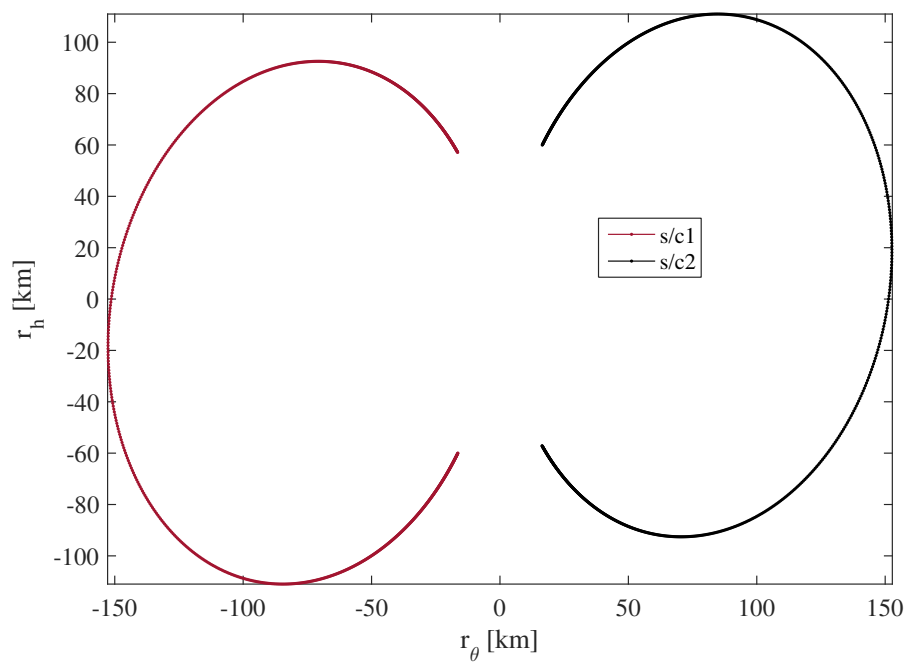

Figure 2: Two spacecraft formation in the $\theta-h$ plane.

\subsection{Inter-spacecraft Measurements}

The set of inter-spacecraft measurements is represented by the relative position and velocity vectors between two spacecraft in the formation expressed as modulus, local azimuth and elevation angles ((Vetrisano \& Vasile , 2016)). 
The observation equation for the position vector is given by:

$$
\mathbf{z}_{r}=h_{r}\left(\mathbf{r}_{i}, \mathbf{r}_{j}\right)=\left[\begin{array}{lll}
d_{r} & \varphi_{r} & \psi_{r}
\end{array}\right]^{T}+\zeta_{r}
$$

where $\zeta_{r}=\left[\zeta_{d_{r}} \zeta_{\varphi_{r}} \zeta_{\psi_{r}}\right]^{T}$ is the measurement noise. This simple measurement is providing the complete spacecraft-to-spacecraft vector in inertial space. Given spacecraft with state $\mathbf{r}_{i}$ and spacecraft with state $\mathbf{r}_{j}$ the spacecraftto-spacecraft vector is $\Delta \mathbf{r}_{i j}=\left[d_{r} \cos \psi_{r} \cos \phi_{r}, d_{r} \sin \psi_{r} \cos \phi_{r}, d_{r} \sin \phi_{r},\right]_{i j}^{T}$. In the case where the relative velocity is also computed, the measurement vector in equation (2) is extended by the relative velocity vector:

$$
\mathbf{z}_{\dot{r}}=h_{\dot{r}}\left(\dot{\mathbf{r}}_{i}, \dot{\mathbf{r}}_{j}\right)=\left[\begin{array}{lll}
d_{v} & \varphi_{v} & \psi_{v}
\end{array}\right]^{T}+\zeta_{v}
$$

where $\zeta_{v}=\left[\zeta_{d_{v}} \zeta_{\varphi_{v}} \zeta_{\psi_{v}}\right]^{T}$ is the measurement noise. The error is due to the attitude error of the spacecraft and to the telemetry error. In the following, the accuracy in the knowledge of the pointing vector is estimated to be between 1e-4 and 1e-3 radians which is compatible with recent advances in attitude sensors for Cubesats (see Dzamba et al. (2014)). Indeed this is only part of the error in the reconstruction of the relative position and velocity vectors. In this paper, however, as working assumption, we consider only the attitude error and we will provide a trend curve that shows the increase in the estimation of the state of the spacecraft as the error in the relative position and velocity vectors increases.

\subsection{Time Of Arrival and Time Difference Of Arrival}

For a source with coordinates $\mathbf{s}_{j}$ and a receiver with coordinates $\mathbf{r}_{1}$ the Time Of Arrival can be written as:

$$
\left(t_{1}-t_{j}\right)=\frac{1}{c} \sqrt{\left(\mathbf{r}_{1}-\mathbf{s}_{j}\right)^{T}\left(\mathbf{r}_{1}-\mathbf{s}_{j}\right)}
$$

and if one squares both sides:

$$
\left(t_{1}-t_{j}\right)^{2}=\frac{1}{c^{2}}\left(\mathbf{r}_{1}^{T} \mathbf{r}_{1}-\mathbf{s}_{j}^{T} \mathbf{r}_{1}-\mathbf{r}_{1}^{T} \mathbf{s}_{j}+\mathbf{s}_{j}^{T} \mathbf{s}_{j}\right)
$$

If the same signal from $s_{j}$ is received by a second spacecraft $\mathbf{r}_{2}$ we have:

$$
\left(t_{2}-t_{j}\right)^{2}=\frac{1}{c^{2}}\left(\mathbf{r}_{2}^{T} \mathbf{r}_{2}-\mathbf{s}_{j}^{T} \mathbf{r}_{2}-\mathbf{r}_{2}^{T} \mathbf{s}_{j}+\mathbf{s}_{j}^{T} \mathbf{s}_{j}\right)
$$


ans by subtracting (6) from (5) one gets the TDOA expression:

$$
\left(t_{2}-t_{j}\right)^{2}-\left(t_{1}-t_{j}\right)^{2}=\frac{1}{c^{2}}\left(\mathbf{r}_{1}^{T} \mathbf{r}_{1}-\mathbf{r}_{2}^{T} \mathbf{r}_{2}-\mathbf{s}_{j}^{T} \mathbf{r}_{1}+\mathbf{s}_{j}^{T} \mathbf{r}_{2}-\mathbf{r}_{1}^{T} \mathbf{s}_{j}+\mathbf{r}_{2}^{T} \mathbf{s}_{j}\right)
$$

where we have six unknowns. The inter-satellite link provides three equations that relate $\mathbf{r}_{1}$ to $\mathbf{r}_{2}$, therefore, the number of unknowns are reduced to three. Thus, with only two receivers and one beacon the problem cannot be completely solved and additional conditions are required. If one more stations is used as a beacon, then the problem becomes completely solvable. Likewise if we had three receivers then the unknowns would be 9 , the inter-satellite link would provide 6 equations and the TOA (or TDOA) other 3. In this case the problem can also be completely solved.

\subsection{Frequency Of Arrival}

If the receiver on spacecraft 1 can measure the Doppler shift we can say that:

$$
\Delta f_{1} \frac{c}{f_{0}}=\frac{\left(\mathbf{r}_{1}-\mathbf{s}_{j}\right)^{T}\left(\dot{\mathbf{r}}_{1}-\dot{\mathbf{s}}_{j}\right)}{r_{1}}
$$

which introduces 3 additional unknowns. If one had two stations and two spacecraft then the Doppler equations would be 4 plus 3 equations giving the relative velocity via inter-satellite link. These 7 equations plus the 3 relative positions and 2 equations (7), one per station, allows one to completely solve the problem.

Note that even in this case if more than two stations or more than two spacecraft were available the Doppler shift would provide the velocities of the spacecraft with respect to the station provided that the inter-satellite link was giving the relative velocity among spacecraft.

\subsection{Time and Frequency Error Estimations}

The main problem with the use of the TOA is the drift and stability of the internal clock of the receiver. In a two way communication system this error can be eliminated by synchronisation of the on board and ground clocks. However, in a one way communication solution the spacecraft needs to reset its clock only using the reference signal coming from ground.

Recent advances in technology for cubesats offer chip scale atomic clocks with an Allan Deviation of $8 \mathrm{e}-12$ for a TAU of 100 s and an ageing rate of less that 1e-9 per year (Conklin et al. (2014)). It is interesting to note that a study by Bloch et al. (2002) shows very good performance in space with 
minimum effect of radiations on atomic clocks. Allan Deviation $\sigma_{y}$ introduces a time deviation $\sigma_{x}$ that can be quantified as (see Riley (2008)):

$$
\sigma_{x}=\frac{\tau}{\sqrt{3}} \bmod \sigma_{y}
$$

where $\bmod \sigma_{y}$ is the modified Allan Deviation. This deviation has to be added to the initial error between the terrestrial clock and the on board clock and other two sources of error: the frequency drift $\Delta f$ and ageing rate $D$. The total error is:

$$
\epsilon_{T O A}=\delta t_{0}+\left(\Delta f / f_{0}\right) t+0.5 D t^{2}+\sigma_{x}
$$

It is reasonable to assume that $\delta t_{0}=0$ and there an initial tuning of the clocks once the spacecraft is in orbit so that $\Delta f=0$. However, as the mission progresses, radiations, temperature variability and ageing, plus the contribution of Allan Deviation will progressively increase the time error.

We assume that the formation is able to synchronise their clocks using a standard two way communication system and standard clock synchronisation algorithm, such as Barkley's algorithm or equivalent. This synchronisation however, does not completely remove the error in (10). If the clocks are well characterised, one can assume that the model can be used to filter the drift and correct the TOA. Although this is a reasonable assumption here we provide also a reset mechanism to partially synchronise the clocks on board with the source.

The idea is based on a heart-beat concept in which the receiver compares the local time increments with the reference time increment transmitted by the ground station. If one calls $t_{0}$ the exact universal time of the ground station and $t_{l}$ the local time on the spacecraft, the spacecraft is expected to receive a first pulse at time $t_{l 1}$ corresponding to a transmission time $t_{01}$. After a time interval $\Delta t_{0}$ the ground station sends a second pulse. When the pulse is received the spacecraft register the time $t_{l 2}$ such that:

$$
t_{l 2}=t_{l 1}+\Delta t_{0}+\frac{\Delta r}{c}+\epsilon_{T O A}
$$

where $\Delta t_{l}=t_{l 2}-t_{l 1}$ is the Time Difference Of Arrival at the same spacecraft, $\Delta r$ is the displacement in the direction of propagation of the radio wave, and $\epsilon_{T O A}$ is the accumulated error of the clock on board. If the frequency at which the two clocks are synchronised is high, $\Delta t_{0}$ is small and one can assume a linear model for the displacement such that:

$$
\Delta t_{l}=\Delta t_{0}+\frac{\dot{r} \Delta t_{0}}{c}+\epsilon_{T O A}
$$


The local velocity can be derived from the Frequency Of Arrival:

$$
\dot{r}=\frac{\Delta f c}{f_{0}}+\epsilon_{F O A}
$$

From which one has that:

$$
\epsilon_{T O A}=\Delta t_{l}-\Delta t_{0}-\frac{\Delta f \Delta t_{0}}{f_{0}}-\frac{\epsilon_{F O A} \Delta t_{0}}{c}
$$

The error in velocity $\epsilon_{F O A}$ depends on the error in the Doppler shift measurement. This error depends on the observation time $T_{o}$, and can be approximated to be $1 / T_{o}$. In order to remove the drift accumulated in the time interval $\Delta t_{0}$ the ratio $\epsilon_{F O A} / c$ has to be smaller than the expected drift of the clock. For a standard clock the expected drift is 1e-6 per second, which gives a required accuracy of the Doppler measurement of less than $300 \mathrm{~m} / \mathrm{s}$ or $12 \mathrm{kHz}$ assuming a carrier in $\mathrm{X}$ band at $12 \mathrm{GHz}$. This accuracy only requires an observation time of $8.3276 \mathrm{e}-5 \mathrm{~s}$. Having said that, it is desirable to reduce the error in TOA down to less than a millisecond, as will be shown later. In this case we can impose a Doppler error of $1 \mathrm{~m} / \mathrm{s}$ that corresponds to $40 \mathrm{~Hz}$ and an observation time of $2.42 \mathrm{e}-2 \mathrm{~s}$.

Note that additional corrections are required to account for the acceleration acting on the spacecraft that has an impact on $\Delta r$. For example at the Earth-Moon distance the acceleration on the spacecraft introduces a difference in $\Delta r$ of about $1.3 \mathrm{e}-4 \mathrm{~m}$ over $10 \mathrm{~s}$ and a prediction error of $4.5 \mathrm{e}-13 \mathrm{~s}$. Furthermore, this approach requires constant communication with the station. In the case of a black-out this system is not sufficient and a model of the clock is required.

In the following the assumption is that once this filtering of the time error is applied the only residual error is Allan Deviation with a resulting range between 1e-8s and 1e-7s depending on the quality of the clock. In the following we will not consider possible relativistic effects and we assume that a correction for atmospheric disturbances is added to the ground transmission.

\section{Analytical Solution of the Orbit Determination Problem}

In this section we derive an analytical solution to the problem of determining the position and velocity of the spacecraft in the formation by using TOA and FOA measurements only. A separate derivation will be presented for the three-spacecraft one-station and for the two-spacecraft two-station case. 


\subsection{Case 1: 3 spacecraft 1 station}

We consider the case in which a single station with coordinates $\mathbf{s}=$ $\left[s_{x}, s_{y}, s_{z}\right]^{T}$ is broadcasting a signal within a cone with aperture $\beta_{s}$. The signal contains the time at which the signal was generated, the coordinates of the station and the direction of the boresight of the station in inertial space. Three spacecraft receive the signal at times $t_{1}, t_{2}$ and $t_{3}$. Spacecraft 1 is identified by the vector $\mathbf{r}_{1}=\left[x_{1}, y_{1}, z_{1}\right]^{T}$ and the relative position vectors with respect to spacecraft 2 and 3 are $\Delta \mathbf{r}_{12}=\left[x_{12}, y_{12}, z_{12}\right]^{T}$ and $\Delta \mathbf{r}_{13}=\left[x_{13}, y_{13}, z_{13}\right]^{T}$. The TOA for the three spacecraft can be written in the following way:

$$
\begin{aligned}
& R_{1}=c^{2}\left(t_{1}-t_{0}\right)^{2}=\left(x_{1}-s_{x}\right)^{2}+\left(y_{1}-s_{y}\right)^{2}+\left(z_{1}-s_{z}\right)^{2} \\
& R_{2}=c^{2}\left(t_{2}-t_{0}\right)^{2}=\left(x_{1}-s_{x}+x_{12}\right)^{2}+\left(y_{1}-s_{y}+y_{12}\right)^{2}+\left(z_{1}-s_{z}+z_{12}\right)^{2} \\
& R_{3}=c^{2}\left(t_{3}-t_{0}\right)^{2}=\left(x_{1}-s_{x}+x_{13}\right)^{2}+\left(y_{1}-s_{y}+y_{13}\right)^{2}+\left(z_{1}-s_{z}+z_{13}\right)^{2}
\end{aligned}
$$

Before proceeding, it is convenient to use the transformed position vector $\mathbf{r}_{1}^{*}=\mathbf{r}_{1}-\mathbf{s}$. After replacing $\mathbf{r}_{1}^{*}$ in Eqs. (15) and developing the last two equations one can write:

$$
\begin{aligned}
& R_{2}-R_{1}-R_{12}=2\left(x_{1}^{*} x_{12}+y_{1}^{*} y_{12}+z_{1}^{*} z_{12}\right) \\
& R_{3}-R_{1}-R_{13}=2\left(x_{1}^{*} x_{13}+y_{1}^{*} y_{13}+z_{1}^{*} z_{13}\right)
\end{aligned}
$$

where $R_{12}=x_{12}^{2}+y_{12}^{2}+z_{12}^{2}$ and $R_{13}=x_{13}^{2}+y_{13}^{2}+z_{13}^{2}$. It is worth noting, at this point, that, in virtue of Eqs. (16) the systematic error in the TOA of spacecraft 2 and 3 is automatically filtered out. If $x_{12}$ and $x_{13}$ are not zero, by multiplying the first equation in (16) by $x_{13}$ and the second by $-x_{12}$ one can solve with respect to $y_{1}^{*}$ to get:

$$
\Delta_{1}=\left(R_{2}-R_{12}-R_{1}\right) x_{13}-\left(R_{3}-R_{13}-R_{1}\right) x_{12}
$$

and

$$
y_{1}^{*}=\frac{\Delta_{1}-b_{1} z_{1}^{*}}{a_{1}}
$$

with $a_{1}=2\left(y_{12} x_{13}-y_{13} x_{12}\right)$ and $b_{1}=2\left(z_{12} x_{13}-z_{13} x_{12}\right)$. Expression (18) can now be inserted into the first of Eqs. (16) to derive an expression for $x_{1}^{*}$ :

$$
x_{1}^{*}=\frac{\Delta_{2}-b_{2} z_{1}^{*}}{a_{2}}
$$

with $\Delta_{2}=R_{2}-R_{12}-R_{1}-2 y_{1}^{*} \Delta_{1} / a_{1}, a_{2}=2 x_{1}$ and $b_{2}=\left(2 z_{1}^{*}-2 y_{1}^{*} b_{1} / a_{1}\right)$. Finally if one substitutes the expressions for $y_{1}^{*}$ and $x_{1}^{*}$ into the first of Eqs. (15) 
the problem reduces to the solution of the following second order algebraic equation in $z_{1}^{*}$ :

$$
A z_{1}^{* 2}+B z_{1}^{*}+C=0
$$

with solutions:

$$
z_{1,12}^{*}=\frac{-B \pm \sqrt{B^{2}-4 A C}}{2 A}
$$

and:

$$
\begin{aligned}
& A=1+\frac{b_{2}^{2}}{a_{2}^{2}}+\frac{b_{1}^{2}}{a_{1}^{2}} \\
& B=-2\left(\frac{\Delta_{2} b_{2}}{a_{2}^{2}}+\frac{\Delta_{1} b_{1}}{a_{1}^{2}}\right) \\
& C=\frac{\Delta_{2}^{2}}{a_{2}^{2}}+\frac{\Delta_{1}^{2}}{a_{1}^{2}}-R_{1}
\end{aligned}
$$

The $R_{1}$ term in $C$ introduces the TOA systematic error (drift and ageing) in the estimation of the position vector. However, if four spacecraft were available one could obtain two equations (20) and remove the systematic error in $C$ by subtracting one to the other. In the following we will not consider the case with more than three spacecraft but we will assume that the error in TOA can be reduced down to the time deviation only. If $x_{12}$ and $x_{13}$ are zero, or $x_{1}$ is zero, system of Eqs.(15) can be solved with respect to $x_{1}^{*}$ or $y_{1}^{*}$ leading again to only two solutions. However, there are cases in which system (15) presents more than two solutions and other cases in which only one solution is possible. In general we can prove the following proposition.

Proposition 1. Given system (15), if the TOA and the intersatellite position vector are measured with infinite precision the following statements are true:

1. If $\left\|\Delta \mathbf{r}_{12} \wedge \Delta \mathbf{r}_{13}\right\|>0$ and $B^{2}-4 A C>0$, system of equations Eqs.(15) has two distinct solutions.

2. If $\left\|\Delta \mathbf{r}_{12} \wedge \Delta \mathbf{r}_{13}\right\|>0$ and $B^{2}-4 A C=0$, system of equations Eqs.(15) has one solution.

3. If $\left\|\Delta \mathbf{r}_{12} \wedge \Delta \mathbf{r}_{13}\right\|=0$, system of equations Eqs.(15) has no unique solution and the locus of all possible solutions is a cylinder.

4. If all spacecraft are aligned with the boresight of the station, system of equations Eqs.(15) has one degenerate solution. 
Proof. System (15) can be rewritten in the following compact form:

$$
\left\{\begin{array}{l}
\mathbf{r}_{1}^{T} \mathbf{r}_{1}=R_{1} ; \\
\mathbf{r}_{2}^{T} \mathbf{r}_{2}=R_{2} ; \\
\mathbf{r}_{3}^{T} \mathbf{r}_{3}=R_{3} ; \\
\Delta \mathbf{r}_{21}=\mathbf{r}_{2}-\mathbf{r}_{1} ; \\
\Delta \mathbf{r}_{31}=\mathbf{r}_{3}-\mathbf{r}_{1} ;
\end{array}\right.
$$

where the first three equations are the spacecraft TOAs and the last two are the relative position vectors provided by the inter-spacecraft link. By inserting the last two ones into the first three, one can obtain the reduced system in $\mathbf{r}_{1}$ :

$$
\left\{\begin{array}{l}
\mathbf{r}_{1}^{T} \mathbf{r}_{1}=R_{1} ; \\
\Delta \mathbf{r}_{21}^{T} \mathbf{r}_{1}=\Gamma_{21} ; \\
\Delta \mathbf{r}_{31}^{T} \mathbf{r}_{1}=\Gamma_{31} ;
\end{array}\right.
$$

with $\Gamma_{21}=\left(R_{2}-R_{1}-D_{21}^{2}\right) / 2, \Gamma_{31}=\left(R_{3}-R_{1}-D_{31}^{2}\right) / 2, D_{21}=\Delta \mathbf{r}_{21}^{T} \Delta \mathbf{r}_{21}$ and $D_{31}=\Delta \mathbf{r}_{31}^{T} \Delta \mathbf{r}_{31}$.

System of Eqs. (24) represents the intersection between two planes and a sphere that, in general, would have two distinct solutions or a unique one when the straight line identified by the two planes is tangent to the sphere (see Fig.3). When $\left\|\Delta \mathbf{r}_{12} \wedge \Delta \mathbf{r}_{13}\right\|=0$ the spacecraft in the formation are aligned along a straight line, and the left hand sides of the last two equations in (24) are proportional to each other. In particular, if one calls $\mathbf{u}=\Delta \mathbf{r}_{21} /|| \Delta \mathbf{r}_{21} \|$ the unit vector in the direction of $\Delta \mathbf{r}_{21}$, then one can reduce the probelm to a single equation:

$$
\mathbf{u}^{T} \mathbf{r}_{1}=\frac{\Gamma_{21}}{D_{21}}
$$

Eq. (25)describes a cylinder whose axis is aligned with $\Delta \mathbf{r}_{12}$ and with radius:

$$
R_{c y l}=r_{1} \sin \alpha ; \cos \alpha=\frac{\Gamma_{21}}{r_{1} D_{21}}
$$

When $\alpha=0$, the cylinder degenerates, the formation is oriented along the boresight of the ground station and a unique solution exists. 


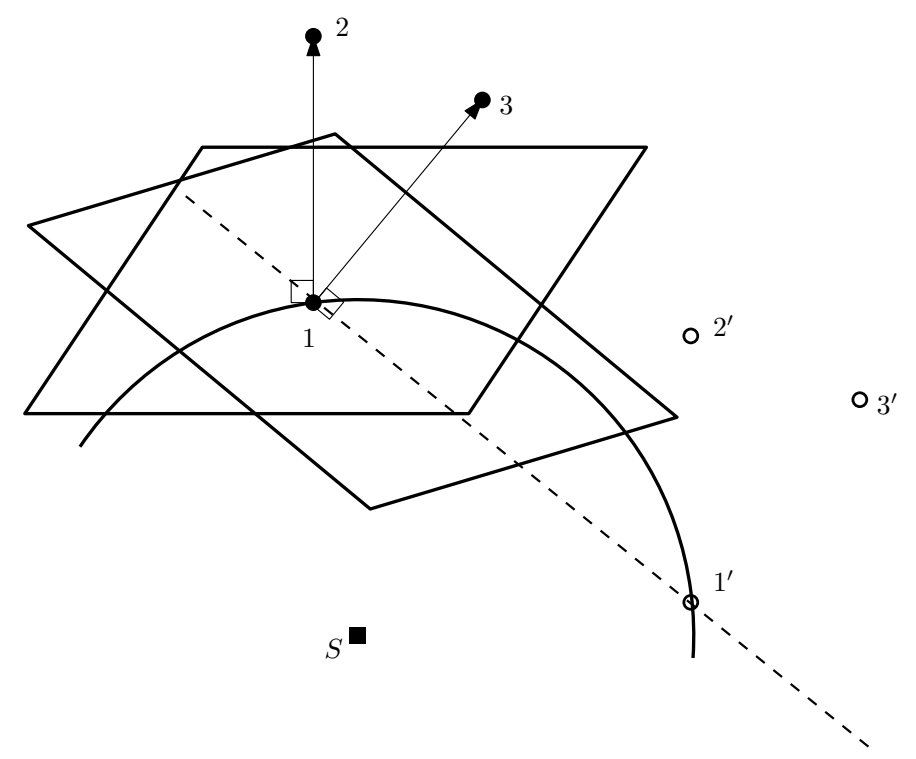

Figure 3: General case for the configuration with spacecraft 1,2, 3 and 1 station $S: 2$ solutions. It can degenerate into a single one when the two points merge or into an infinite set when the two planes overlap and the solutions form a circle.

When two distinct solutions exist, only one has to be considered. Given that the information on the aperture of the visibility cone of the antenna is transmitted with the signal, a simple discrimination criterion is to select the position vector whose angle with respect to the boresight of the antenna is smaller than the aperture of the visibility cone. A further piece of information like the direction of the Sun can be used as an additional criterion to discriminate the correct solution.

Once the full position vector $\left[x_{1}^{*}, y_{1}^{*}, z_{1}^{*}\right]^{T}$ is available one can compute the velocity vector starting from the following system that makes use of Doppler measurements (or FOA):

$$
\begin{aligned}
& V_{1}=c \frac{\Delta f_{01}}{f_{0}} \sqrt{R_{1}}=x_{1}^{*} v_{x_{1}}+y_{1}^{*} v_{y_{1}}+z_{1}^{*} v_{z_{1}} \\
& V_{2}=c \frac{\Delta f_{02}}{f_{0}} \sqrt{R_{2}}=\left(x_{1}^{*}+x_{12}\right)\left(v_{x_{1}}+\Delta v_{x_{12}}\right)+\left(y_{1}^{*}+y_{12}\right)\left(v_{y_{1}}+\Delta v_{y_{12}}\right)+ \\
& \left(z_{1}^{*}+z_{12}\right)\left(v_{z_{1}}+\Delta v_{z_{12}}\right) \\
& V_{3}=c \frac{\Delta f_{03}}{f_{0}} \sqrt{R_{3}}=\left(x_{1}^{*}+x_{13}\right)\left(v_{x_{1}}+\Delta v_{x_{13}}\right)+\left(y_{1}^{*}+y_{13}\right)\left(v_{y_{1}}+\Delta v_{y_{13}}\right)+ \\
& \left(z_{1}^{*}+z_{13}\right)\left(v_{z_{1}}+\Delta v_{z_{13}}\right)
\end{aligned}
$$

where $\Delta \mathbf{v}_{12}$ and $\Delta \mathbf{v}_{13}$ are the measured relative velocities of spacecraft 2 and 3 with respect to spacecraft 1 respectively. The velocity vector $\mathbf{v}_{1}$ is 
calculated with respect to the ground station and includes the rotation of the Earth. System (27) is linear in the velocity vector $\mathbf{v}_{1}$ and can be solved provided that the matrix:

$$
\left[\begin{array}{ccc}
x_{1}^{*} & y_{1}^{*} & z_{1}^{*} \\
x_{1}^{*}+x_{12} & y_{1}^{*}+y_{12} & z_{1}^{*}+z_{12} \\
x_{1}^{*}+x_{13} & y_{1}^{*}+y_{13} & z_{1}^{*}+z_{13}
\end{array}\right]
$$

is full rank, which implies that the three position vectors of the three spacecraft should not be pairwise aligned.

\subsection{Case 2: 2 spacecraft 2 stations}

In the case of only two spacecraft, the time of arrival from a single station is not sufficient to determine the complete position vector. A complete solution can be computed by adding a second emitting station, synchronized with the first one. Given stations with known inertial coordinates $\mathbf{s}_{1}=\left[s_{x_{1}}, s_{y_{1}}, s_{z_{1}}\right]^{T}$ and $\mathbf{s}_{2}=\left[s_{x_{2}}, s_{y_{2}}, s_{z_{2}}\right]^{T}$ and spacecraft with inertial coordinates $\mathbf{r}_{1}=\left[x_{1}, y_{1}, z_{1}\right]^{T}$ and $\mathbf{r}_{2}=\left[x_{2}, y_{2}, z_{2}\right]^{T}$, it is assumed that the signals from station 1 and 2 are received by spacecraft 1 at times $t_{1}$ and $t_{2}$ while spacecraft 2 received the signal from station 2 at time $t_{3}$. With this configuration, the following system needs to be solved to get the position vector of spacecraft 1:

$$
\begin{aligned}
& R_{11}=c^{2}\left(t_{1}-t_{0}\right)^{2}=\left(x_{1}-s_{x_{1}}\right)^{2}+\left(y_{1}-s_{y_{1}}\right)^{2}+\left(z_{1}-s_{z_{1}}\right)^{2} \\
& R_{12}=c^{2}\left(t_{2}-t_{0}\right)^{2}=\left(x_{1}-s_{x_{2}}\right)^{2}+\left(y_{1}-s_{y_{2}}\right)^{2}+\left(z_{1}-s_{z_{2}}\right)^{2} \\
& R_{21}=c^{2}\left(t_{3}-t_{0}\right)^{2}=\left(x_{1}+x_{12}-s_{x_{1}}\right)^{2}+\left(y_{1}+y_{12}-s_{y_{1}}\right)^{2}+\left(z_{1}+y_{12}-s_{z_{1}}\right)^{2}
\end{aligned}
$$

Before proceeding, it is convenient to use the transformed position vector $\mathbf{r}_{1}^{*}=\mathbf{r}_{1}-\mathbf{s}_{1}$ and $\mathbf{s}_{2}^{*}=\mathbf{s}_{2}-\mathbf{s}_{1}$. After substituting vectors $\mathbf{r}_{1}^{*}$ and $\mathbf{s}_{2}^{*}$ into (29) and developing the last two equations, one gets:

$$
\begin{aligned}
& R_{12}-R_{11}-R_{S 2}=-\left(x_{1}^{*} s_{x_{2}}^{*}+y_{1}^{*} s_{y_{2}}^{*}+z_{1} s_{z_{2}}^{*}\right) \\
& R_{21}-R_{11}-\Delta r_{12}=2\left(x_{1}^{*} x_{12}+y_{1}^{*} y_{12}+z_{1}^{*} z_{12}\right)
\end{aligned}
$$

where $R_{S 2}=s_{x_{2}}^{* 2}+s_{y_{2}}^{* 2}+s_{z_{2}}^{* 2}$ and $\Delta r_{12}=x_{12}^{2}+y_{12}^{2}+z_{12}^{2}$. If $x_{12}$ and $x_{S 2}$ are not zero, by multiplying the first equation in (30) by $s_{x_{2}}^{*}$ and the second by $x_{12}$ one can solve with respect to $y_{1}^{*}$ to get:

$$
y_{1}^{*}=\frac{\Delta_{1}-b_{1} z_{1}^{*}}{a_{1}}
$$


where $a_{1}=2\left(y_{12} s_{x_{2}}^{*}-s_{y_{2}}^{*} x_{12}\right), b_{1}=2\left(z_{12} s_{x_{2}}^{*}-s_{z_{2}}^{*} x_{12}\right)$ and $\Delta_{1}=2\left(R_{12}-R_{11}-\right.$ $\left.R_{S 2}\right) x_{12}+\left(R_{21}-R_{11}-\Delta r_{12}\right) s_{x_{2}}^{*}$. By substituting $y_{1}^{*}$ in the first of (30) and solving for $x_{1}^{*}$ we get:

$$
x_{1}^{*}=\frac{\Delta_{2}-b_{2} z_{1}^{*}}{a_{2}}
$$

where $a_{2}=2 x_{12}, b_{2}=2\left(z_{12}-y_{12} b_{1} / a_{1}\right)$ and $\Delta_{2}=R_{21}-R_{11}-\Delta r_{12}-$ $2 y_{12} \Delta_{1} / a_{1}$; By substituting the values of $x_{1}^{*}$ and $y_{1}^{*}$ in (29) we reduce, again, the problem to a second order algebraic equation in $z_{1}^{*}$ with solutions:

$$
z_{1,12}^{*}=\frac{-B \pm \sqrt{B^{2}-4 A C}}{2 A}
$$

with the coefficients $A, B$ and $C$ that have the same form as in (22):

$$
\begin{aligned}
& A=1+b_{2}^{2} / a_{2}^{2}+b_{1}^{2} / a_{1}^{2} \\
& B=-2\left(\Delta_{2} b_{2} / a_{2}^{2}+\Delta_{1} b_{1} / a_{1}^{2}\right) \\
& C=\Delta_{2}^{2} / a_{2}^{2}+\Delta_{1}^{2} / a_{1}^{2}-R_{11}
\end{aligned}
$$

Even in this case $C$ contains the systematic error in TOA, which could be mitigated by adding a spacecraft or a further TOA equation. As for the case of three spacecraft and one station, one can prove the following proposition that states that in general two solutions exist, except for some specific cases in which infinite solutions or a single solution exist.

Proposition 2. Given system (29), if the TOA and the intersatellite position vector are measured with infinite precision the following statements are true:

1. If $\left\|\Delta \mathbf{r}_{12} \wedge \mathbf{s}_{2}^{*}\right\|>0$ and $B^{2}-4 A C>0$, system of equations Eqs.(29) has two distinct solutions.

2. If $\left\|\Delta \mathbf{r}_{12} \wedge \mathbf{s}_{2}^{*}\right\|>0$ and $B^{2}-4 A C=0$, system of equations Eqs.(29) has one solution.

3. If $\left\|\Delta \mathbf{r}_{12} \wedge \mathbf{s}_{2}^{*}\right\|=0$, system of equations Eqs.(29) has no unique solution and the locus of all possible solutions is a cylinder.

Proof. The proof is analogous to the one for the three spacecraft one station case where one of the two intersatellite vectors is replced by the station-tostation vector .

Once the full position vector $\left[x_{1}^{*}, y_{1}^{*}, z_{1}^{*}\right]^{T}$ is available one can compute the velocity vector starting from the following system that makes use of Doppler 
measurements (or FOA):

$$
\begin{aligned}
& V_{1}=c \frac{\Delta f_{01}}{f_{0}} \sqrt{R_{11}}=x_{1}^{*} v_{x_{1}}+y_{1}^{*} v_{y_{1}}+z_{1}^{*} v_{z_{1}} \\
& V_{2}=c \frac{\Delta f_{02}}{f_{0}} \sqrt{R_{12}}=\left(x_{1}^{*}-s_{x_{2}}^{*}\right) v_{x_{1}}+\left(y_{1}^{*}-s_{y_{2}}^{*}\right) v_{y_{1}}+\left(z_{1}^{*}-s_{z_{2}}^{*}\right) v_{z_{1}} \\
& V_{3}=c \frac{\Delta f_{03}}{f_{0}} \sqrt{R_{22}}=\left(x_{1}^{*}+x_{12}-s_{x_{2}}^{*}\right)\left(v_{x_{1}}+\Delta v_{x_{12}}\right)+ \\
& \left(y_{1}^{*}+y_{12}-s_{y_{2}}^{*}\right)\left(v_{y_{1}}+\Delta v_{y_{12}}\right)+\left(z_{1}^{*}+z_{12}-s_{z_{2}}^{*}\right)\left(v_{z_{1}}+\Delta v_{z_{12}}\right)
\end{aligned}
$$

where $\Delta \mathbf{v}_{12}$ is the measured relative velocity of spacecraft 2 with respect to spacecraft 1 . The velocity vector $\mathbf{v}_{1}$ is calculated with respect to station 1 and includes the rotation of the Earth. Furthermore, it is assumed that the velocity with respect to station 1 is almost the same as the one with respect to station 2. Note that this is not always the case. For example if the two spacecraft were moving in the equatorial plane and the stations were along the equator at different meridians. In this case a spacecraft could see one station moving away while the other is approaching. As it will be shown in the following, this configuration is not ideal as it could lead to an infinite number of solutions. A better configuration is with the two stations along the same meridian. In this case the assumption on the velocities is acceptable. System (35) is once again linear in $\mathbf{v}_{1}$ and can be solved provided that the matrix:

$$
\left[\begin{array}{ccc}
x_{1}^{*} & y_{1}^{*} & z_{1}^{*} \\
\left(x_{1}^{*}-s_{x_{2}}^{*}\right) & \left(y_{1}^{*}-s_{y_{2}}^{*}\right) & \left(z_{1}^{*}-s_{z_{2}}^{*}\right) \\
\left(x_{1}^{*}+x_{12}-s_{x_{2}}^{*}\right) & \left(y_{1}^{*}+y_{12}-s_{y_{2}}^{*}\right) & \left(z_{1}^{*}+z_{12}-s_{z_{2}}^{*}\right)
\end{array}\right]
$$

is full rank, which implies that the position vector of spacecraft 1 with respect to station 1 and 2 and the position vector of spacecraft 2 with respect to station 2 should not be parallel.

\subsection{TDOA Position Error}

The analytical solution is assuming that one can reconstruct the position of one spacecraft given the TOAs at the other spacecraft. If one considers the three-spacecraft case, when spacecraft 2 receives the signal and communicates with spacecraft 1 , spacecraft 1 would have moved by a quantity $\Delta r_{\text {sync }}$. The same would happen when spacecraft 3 receives the signal and communicates with spacecraft 1. System of equations 15 does not include this correction for simplicity of derivation. However, a more precise and correct estimation would need to include $\Delta r_{\text {sync }}$. The error in position estimation however, can be approximated as the local spacecraft velocity times twice the TDOA. This 
error is small compared to the noise in the intersatellite position vector and in the TOA.

\section{Measurement Analysis}

In the previous section we derived an analytical solution for the orbit determination problem and identified the cases in which the system is not observable. The derivation assumed perfect measurements and infinite numerical precision. On the other hand it is desirable to quantify the precision of the OD solution when finite precision is considered and measurement errors are introduced in the measurement chain.

In this section we present the results of a number of numerical simulations in which the error in the estimation of position and velocity is quantified as a function of the error in the measurements and of some geometric quantities, in particular the relative position of the spacecraft and the stations.

\subsection{Case 1: three-spacecraft one-station}

We first analyse the dependency of the estimation error on the numerical accuracy as the distance from the station increases. We call this error, parallax error. In order to do so we increase the apogee of the reference orbit and keep the maximum relative distance of the spacecraft in the formation constant. The simulations were run on an Intel Core i7, in Matlab with 64 bit precision. The resulting position error of spacecraft 1 with respect to the Earth centered reference frame can be seen in Fig.4a for the nominal case of the transfer up to the Moon distance. We consider only part of the transfer orbit from a true anomaly between 135 and 225 degrees. Fig.4b shows the estimation error but for an apogee 100 times higher. As one can see the peak error increases by 4 orders of magnitude.

This is a numerical error that could be mitigated by a rescaling of the equations, note, however, that the error remains small compared to the error introduced by TOA, intersatellite link and FOA measurements.

The second set of analyses introduces errors in the TOA, the intersatellite links and FOA. The signal produced by the station is received at three different TOAs by the three spacecraft. The three spacecraft maintain a knowledge of their relative position and local time by inter-satellite link and synchronization of the clocks. In this analysis spacecraft number 1 maintains its clock synchronized with the ground station using the technique presented 


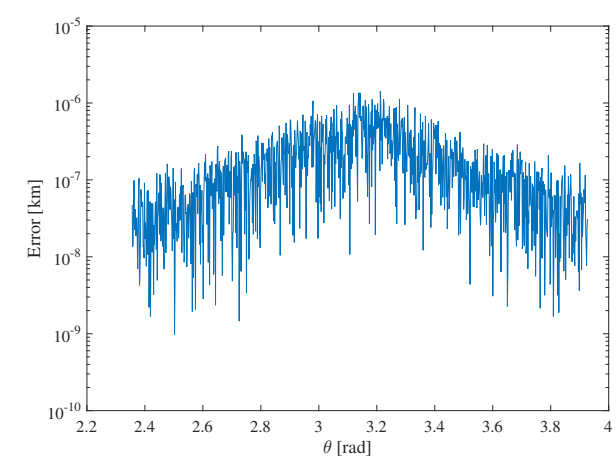

(a)

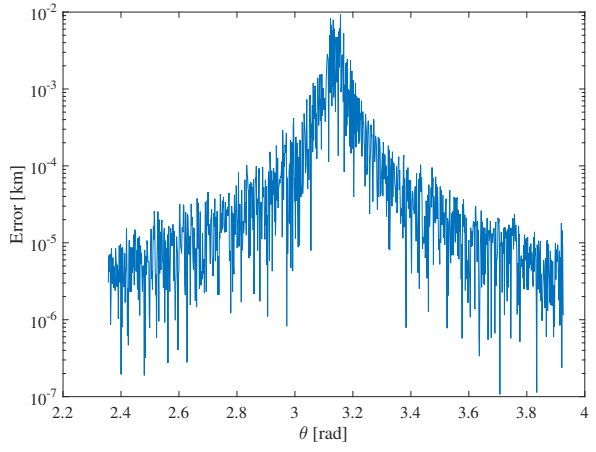

(b)

Figure 4: Case 1 - Estimated position error vs true anomaly: a) nominal reference orbit, b) apogee increased by a factor 100 with constant maximum distance among spacecraft.

in the previous section. Fig. 5a shows the estimation error along the trajectory for an increasing error in the estimation of the intersatellite position vector and TOA noise. The errors $\zeta_{\psi_{r}}, \zeta_{\phi_{r}}, \zeta_{\psi_{v}}$ and $\zeta_{\phi_{v}}$ in the directions of the intersatellite position and velocity vectors range between $1 \mathrm{e}-4$ and 1e3 radians. The relative ranging error is instead between $2 \mathrm{e}-3$ and $2 \mathrm{e}-2 \mathrm{~km}$ and is consistent with COTS qualified components like RelNav (Voronka (2011)). The noise in the measurement of the TOA and, as mentioned before, corresponds only to the time deviation component with a value in the range $[1 e-8,1 e-7] s$. We consider a series of four stations at 45 degrees of latitude north, spaced along the parallel by 90 degrees, so that at least one station is always in view. The aperture of the antenna cone is assumed to be 66.67 degrees which allows for an easy discrimination between the two solutions of the orbit determination problem.

Fig. 5b shows the position error due to the noise in the TOA without any intersatellite link noise. Taking the velocity error from RelNav, the error on the measured radial velocity is in the interval $[1 \mathrm{e}-6 \mathrm{e}-6] \mathrm{km} / \mathrm{s}$ for both the intersatellite link and the Doppler from the ground station.

Fig. 6a and $6 \mathrm{~b}$ show, respectively, the velocity error due to the combination of pointing error, TOA and FOA noise, and the error due to TOA and FOA noise only.

Figure 7 shows the maximum error in position and velocity along the transfer for different magnitudes of the values of $\zeta_{\psi_{r}}, \zeta_{\phi_{r}}, \zeta_{\psi_{v}}$ and $\zeta_{\phi_{v}}$. The maximum error is calculated over the output of 100 simulations. All the 


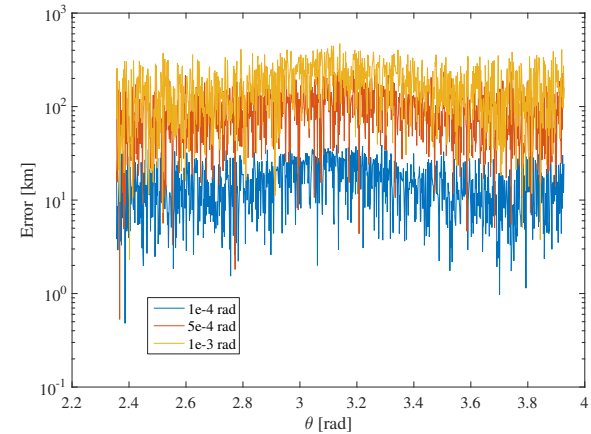

(a)

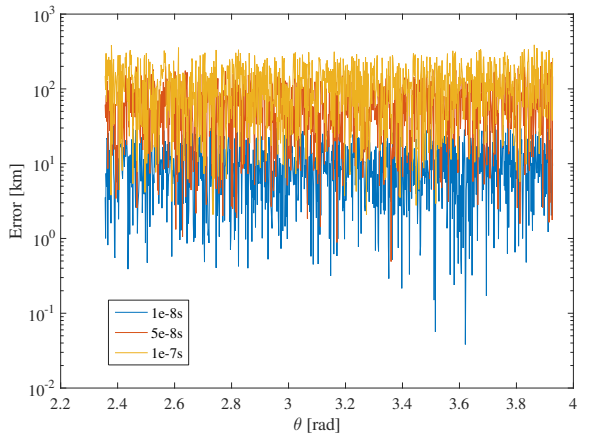

(b)

Figure 5: Case 1 - Position error along the Earth-Moon transfer:a) pointing accuracy error between 1e-4 and 1e-3 radians, b) TOA relative error between 1e-8s and 1e-7s

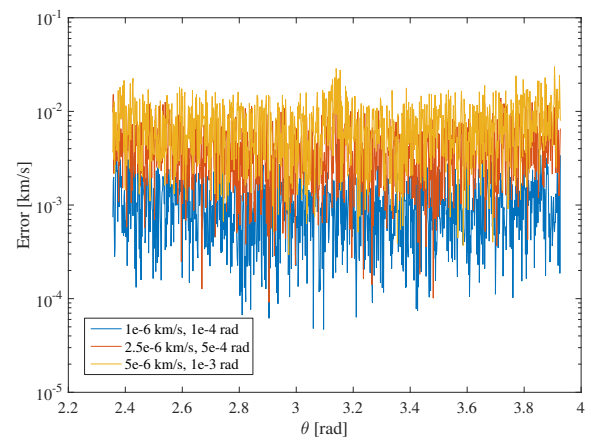

(a)

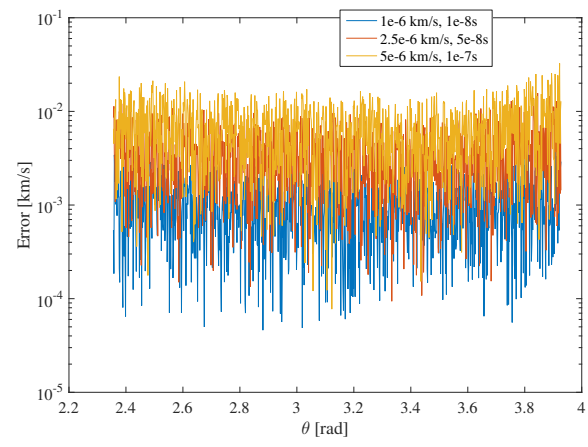

(b)

Figure 6: Case 1 - Velocity error along the Earth-Moon transfer considering: a) a pointing accuracy error between 1e-4 and 1e-3 radians, b) a TOA relative error between 1e-8s and $1 \mathrm{e}-7 \mathrm{~s}$.

errors are $1-\sigma$.

\subsection{Case 2: two-spacecraft two-station}

Also in this case we first analyse the dependency of the estimation error on the numerical accuracy as the distance from the stations increases. As in the previous case we increase the apogee of the reference orbit and keep the maximum relative distance of the spacecraft in the formation constant. The resulting position error of spacecraft 1 with respect to the Earth centred 


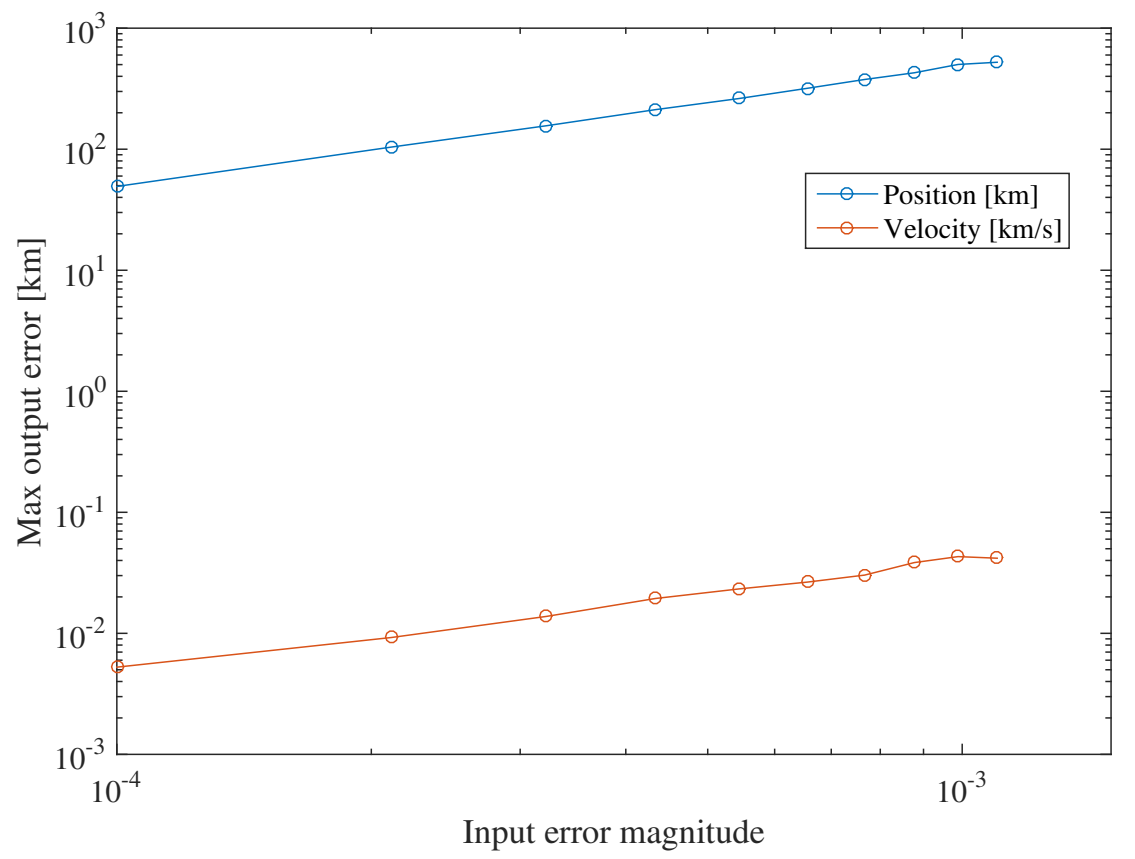

Figure 7: Case 1 - Velocity and position error as a function of the input error magnitude on the pointing accuracy

reference frame can be seen in Fig.8a for the nominal case of a transfer to the Moon distance. Fig.8b shows the estimation error but for an apogee 100 times higher. As one can see the peak error increases, once again, by 4 orders of magnitude.

The second set of analysis introduces errors in the TOA, FOA and intersatellite links. The signals produced by the two stations is received at three different TOAs by the two spacecraft. The two spacecraft maintain a knowledge of their relative position and local time by inter-satellite link and synchronisation of the clocks. In this analysis spacecraft number 1 maintains its clock synchronised with the ground station using the technique presented in the previous section. Fig. 9a shows the estimation error along the trajectory for an increasing error in the estimation of the intersatellite position and velocity vectors assuming a pointing accuracy that ranges between 1e-4 and 1e-3 radians and an increasing TOA error. As for the three-spacecraft case, the relative ranging error is between $2 \mathrm{e}-3$ and $2 \mathrm{e}-2 \mathrm{~km}$ and the noise is given only by the time deviation component with values in the range 


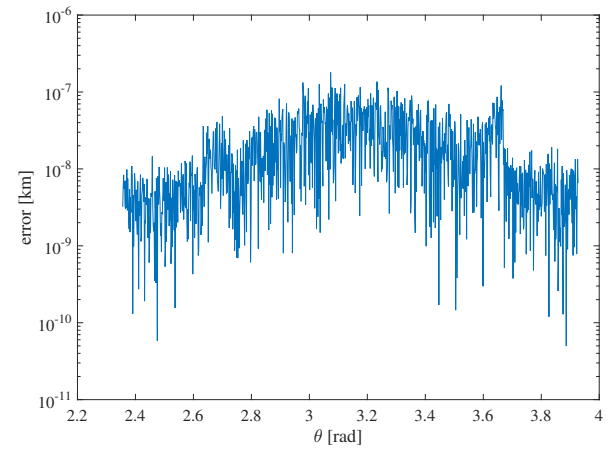

(a)

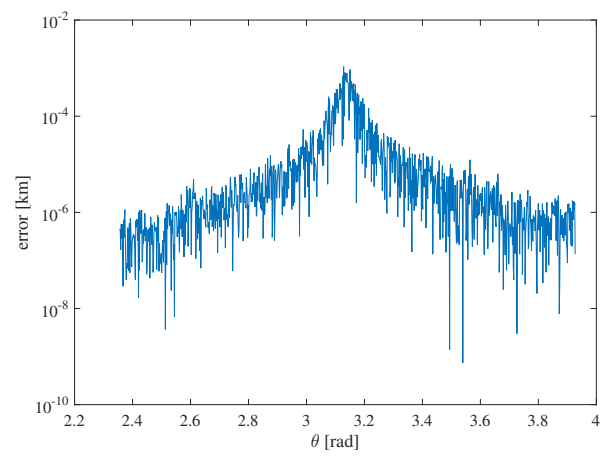

(b)

Figure 8: Case2 - Estimated position error vs true anomaly: a) nominal reference orbit, b) apogee increased by a factor 100

$[1 e-8,1 e-7] s$. Even in this case, we consider a series of four stations at 45 degrees of latitude north, spaced along the parallel by 90 degrees. For each northern station there is a southern station at 15 degrees south. Each pair of northern and souther station is used a beacon for the two-spacecraft two-station case. As before the aperture of the antenna cone is assumed to be 66.67 degrees.

Fig. 9b shows the position error along the trajectory for an increasing noise in the TOA only. For the estimation of the velocity vector the same error, as in the three-spacecraft case, on the measured radial velocity is considered for both the intersatellite link and the Doppler from the ground station. The position of the stations on Earth is expected to be known with infinite precision and their clocks are perfectly synchronised. The location of the second station in the pair is of significant importance for two main reasons: one is the relative velocity with respect to the spacecraft given the approximation introduced in the analytical derivation, the other is the ability to discriminate between the two solutions. We also moved the stations along the same meridian to reduce their relative angular separation down to 1 degree. The effect is similar to the parallax error of the three-spacecraft case but more importantly it becomes more problematic to discriminate between the two solutions unless the cone angle of the station reduces accordingly.

Fig. 10a and 10b show, respectively, the velocity error due to pointing error, TOA and FOA noise, and the velocity error due to FOA and TOA only. Figure 11 shows the maximum error in position and velocity along 


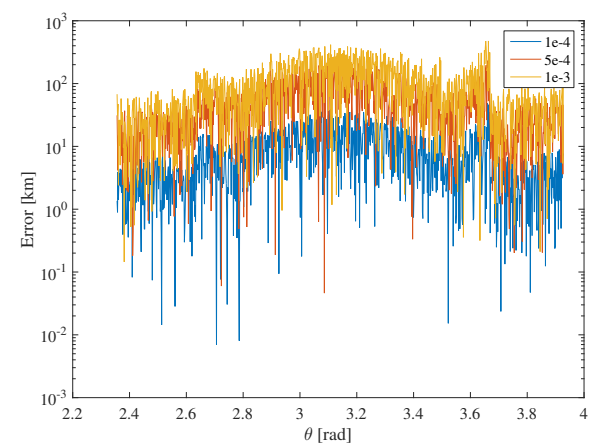

(a)

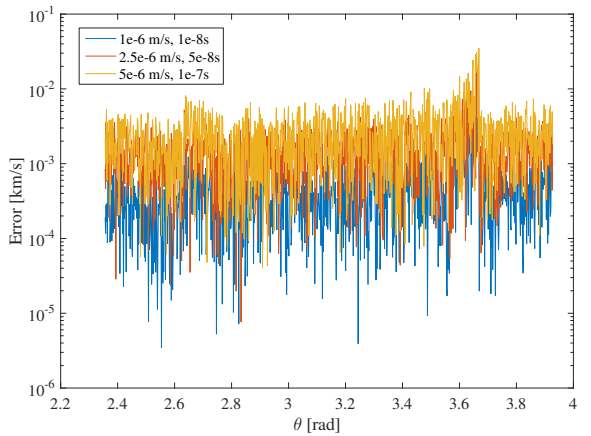

(b)

Figure 9: Case 2 - Position error along the Earth-Moon transfer:a) pointing accuracy error between 1e-4 and 1e-3 radians, b) TOA relative error between 1e-8s and 1e-7s

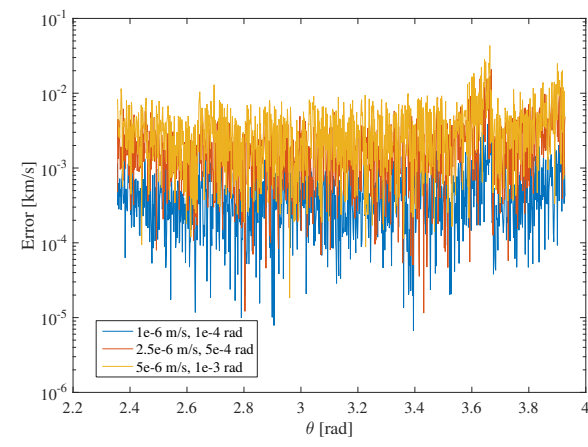

(a)

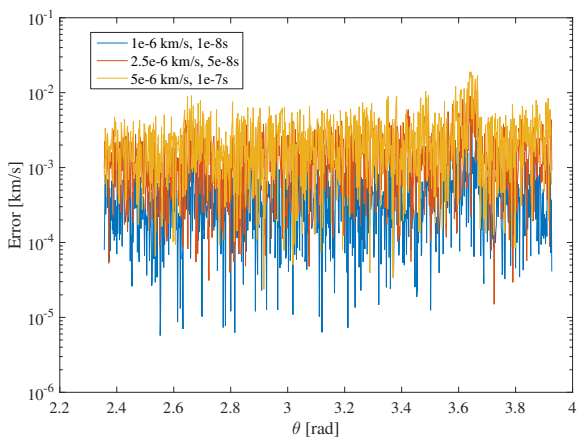

(b)

Figure 10: Case 2 - Velocity error along the Earth-Moon transfer considering: a) a pointing accuracy error between 1e-4 and 1e-3 radians, b) a TOA relative error between 1e-8s and $1 \mathrm{e}-7 \mathrm{~s}$

the transfer for different errors in the magnitude of the pointing accuracy error for Case 2. The maximum error is calculated over the output of 100 simulations. The slope in the loglog plot is about 2.7.

\section{Final Remarks}

The paper presented an analysis of the accuracy in position and velocity estimation for a small formation of CubeSats beyond LEO. The underlying 


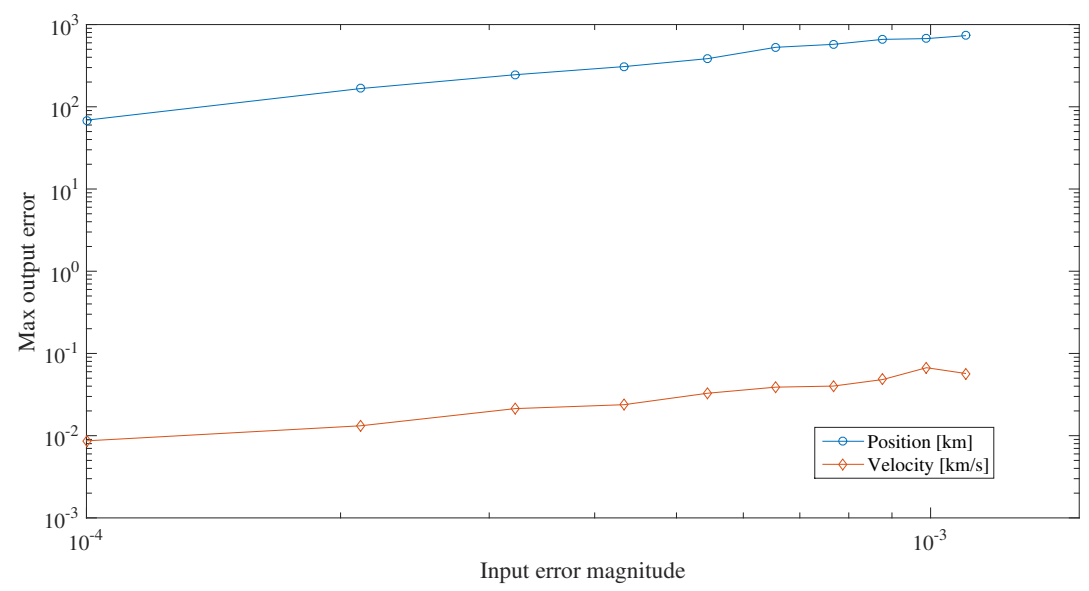

Figure 11: Case 2 - Velocity and position error as a function of the input error magnitude on the pointing accuracy

assumption is that only one way communications from ground station to CubeSat are available on a regular basis and the formation has to estimate their position and velocity autonomously. It is further assumed that occasional communications from the cubesat to the ground station are possible to downlink telemetry. The paper explored different configurations with either three spacecraft and one reference ground station or two spacecraft and two reference ground station.

In both cases an analytical solution was derived to calculate both position and velocity and it was demonstrated that two solutions are generally possible except for some limited cases. In order to discriminate between the two solutions the aperture of the antenna cone can be used although in some cases a third bearing measurement might be required to avoid ambiguity. It was found that both configurations provide similar results. The error in the estimation of the position of the CubeSats is equally affected by the TOA error and the pointing accuracy. The estimation error includes a parallax error, due to numerical accuracy, that grows proportionally to the distance from the source. It was shown that in both cases a few $10 \mathrm{~s}$ of $\mathrm{m} / \mathrm{s}$ accuracy in velocity and hundreds of $\mathrm{km}$ in position is achievable in deep space without any filtering or error model, except for the TOA, if the error in pointing accuracy is limited to 1e-3 radians and the time deviation to 1e-7s. The inclusion of multiple measurements can further improve the estimation of both position and velocity. Future work will be dedicated to incorporate 
this measurement model in a filtering process and extend this techniques to longer distances from the source.

\section{References}

Asmar, S. \& Matousek, S., Mars Cube One (MarCO) - The First Planetary CubeSat Mission, Jet Propulsion Laboratory NASA Report, 2014.

Ho, K.C. \& Chan Y.T. 1997, Geolocation of a Known Altitude Object From TDOA and FDOA Measurements, IEEE Transactions on Aerospace and Electronic Systems, 33, 3, 770-783.

Li, S., Cui, P.Y. \& Cui, H.T. 2007, Vision-aided inertial navigation for pinpoint planetary landing, Aerospace Science and Technology, 11, 6, 499-506.

Mason, J. 2004, Algebraic two-satellite TOA/FOA position solution on an ellipsoidal Earth, IEEE Transactions on Aerospace and Electronic Systems, $40,3,1087-1092$.

Musicki, D. \& Koch, W., Geolocation using TDOA and FDOA measurements, In Proceedings of the 11th International Conference on Information Fusion, 2008.

Torrieri, D.J. 1984, Statistical Theory of Passive Location Systems, IEEE Transactions on Aerospace and Electronic Systems, 20, 2, 183-198.

Ulman, R.J. 2001, Motion detection using TDOA and FDOA measurements, IEEE Transactions on Aerospace and Electronic Systems, 37, 2, 759-764.

Vetrisano, M. \& Vasile, M. 2016, Autonomous Navigation of a Spacecraft Formation in the Proximity of an Asteroid, Advances in Space Research, $57,8,1783-1804$.

Dzamba, T., Enright, J., Sinclair, D., Amankawah, K., Votel, R., Javanovic, I., McVittie, G., Success by 1000 Improvements: Flight Qualification of the ST-16 Star Tracker. SSC14-XII-1, 28th Annual AIAA/USU Conference on Small Satellites. .

Zhang, L., Wang, D. \& Wu, Y. 2014, Performance Analysis of TDOA and FDOA Location by Differential Calibration with Calibration Sources, Journal of Communications, 9, 6, 483-489. 
Conklin, J., Barnwell, N., Caro,L., Carrascilla, M., Formoso, O., Nydam, S., FitzCoy, N. Optical time transfer for future disaggregated small satellite navigation systems. 28th AIAA/USO Conference on Small Satellite, SSC14-XI-5, Logan, UT, USA, 2014.

Bloch, M., Mancini,O., McClelland, T., Performance of Rubidium and Quartz Clocks in Space. IEEE International Frequency Control Symposium and PDA Exhibition, New Orleans, LA, USA, USA, 2002. DOI: 10.1109/FREQ.2002.1075936

Riley, W.J., Handbook of Frequency Stability Analysis. NIST Special Publication 1065, 2008. (http://tf.nist.gov/timefreq/general/pdf/2220.pdf)

Voronka, N., Newton, T., Relative Navigation, Timing \& Data Communications for CubeSat Clusters, 8th Annual CubeSat Developers Workshop, 2011 April 20-22, 2011 Cal Poly State University, San Luis Obispo, California. 